\title{
Will Cannabis or Cannabinoids Protect You from SARS-CoV-2 Infection or Treat COVID-19?
}

\author{
Joshua D. Brown ${ }^{a, b}$ Amie J. Goodin ${ }^{a, b}$ \\ ${ }^{a}$ Consortium for Medical Marijuana Clinical Outcomes Research, University of Florida, Gainesville, FL, USA; \\ ${ }^{b}$ Center for Drug Evaluation and Safety (CoDES), Department of Pharmaceutical Outcomes \& Policy, University of \\ Florida, Gainesville, FL, USA
}

\section{Key Points}

- A recent study reported that two cannabinoids, cannabidiolic acid (CBDA) and cannabigerolic acid (CBGA), could block cellular entry of the virus that causes COVID-19 during in vitro experiments using cell cultures in a laboratory

- There is a low likelihood of translating these preclinical research findings to cannabinoid-based therapies due to clinical and pragmatic concerns with dosing that render CBDA and CBGA (as well as other cannabinoids) to be unlikely candidates for further drug development. These include, for example, a short half-life of CBDA, requiring frequent dosing intervals; high doses required at each interval to match the inhibitory concentrations studied; and high cost and lack of availability of CBDA and CBGA.

- Replicating the observed effects in the complex human body is unlikely due to the interplay of these compounds within the endocannabinoid system, and there are known and hypothesized safety concerns for the doses required.

- Cannabinoids, including CBDA and CBGA, are not recommended for the treatment or prevention of SARS-CoV-2 infection.

- Recreational or medical use of currently available cannabis-derived products are at doses much lower than those studied and are unlikely to provide any benefit against SARS-CoV-2 infection.

\section{Keywords}

COVID-19 · SARS-CoV-2 · Cannabis and COVID-19 .

Cannabinoids and COVID-19 - Medical cannabis .

Medical marijuana · Cannabidiol · Cannabidiol .

Tetrahydrocannabinol · Drug repurposing

\section{Commentary}

A study recently published in the Journal of Natural Products garnered significant media attention and generated headlines such as "COVID-19 blocked by cannabis compounds," "Can weed cure COVID?," and "Compounds in hemp can help control COVID-19 spread." The study by van Breemen et al. [1] used bioanalytical methods and human cell cultures to evaluate whether four cannabinoids could block cellular entry of the virus that causes COVID-19. They report that two cannabinoids, cannabidiolic acid (CBDA) and cannabigerolic acid (CBGA), were effective against cellular infection by SARS-CoV-2 at 24 and $37 \mu \mathrm{g} / \mathrm{mL}$, respectively. The authors conclude that these cannabinoids have a history of safe human use and have the potential to prevent and treat SARS-CoV-2 infection [1]. (c) 2022 The Author(s)

Published by S. Karger AG, Basel

This is an Open Access article licensed under the Creative Commons Attribution-NonCommercial-4.0 International License (CC BY-NC) (http://www.karger.com/Services/OpenAccessLicense), applicable to the online version of the article only. Usage and distribution for commercial purposes requires written permission.
Correspondence to:

Joshua D. Brown, joshua.brown@ufl.edu 


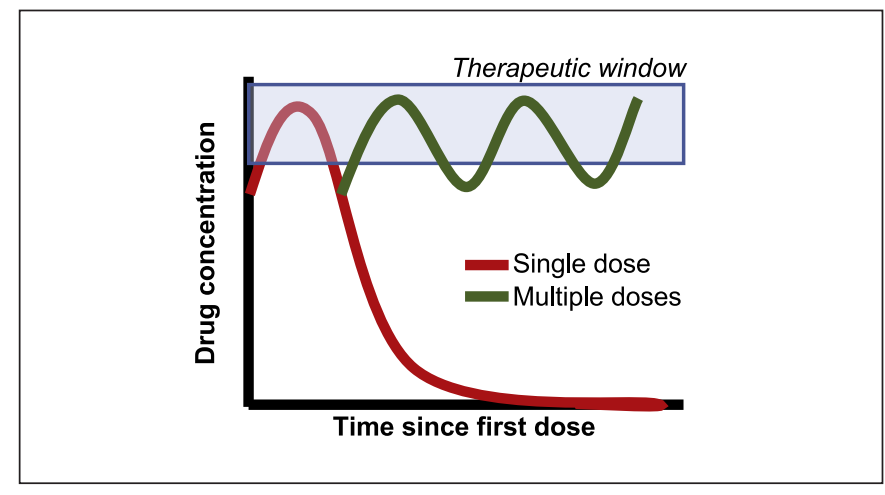

Fig. 1. Hypothetical pharmacokinetic profile of a drug with a therapeutic window. The shaded region is the concentration needed to provide a therapeutic effect, such as blocking viral entry into cells. Due to a short half-life, multiple doses per day of the active cannabinoids identified by the van Breemen study would be needed to reach this concentration. These high doses have never been studied in humans nor are they readily available or affordable.

The promise of "drug repurposing" spurred interest in many compounds as potential COVID-19 treatments; notably, hydroxychloroquine, azithromycin, and ivermectin $[2,3]$. Much of the interest in these compounds was generated from in vitro studies. In vitro studies like these are conducted on human cells developed for research purposes that are incubated in a laboratory, and a cocktail of the compound of interest and virus is introduced within a controlled environment. These approaches are typical of how all medications are developed in the early stages of the drug discovery process, where in vitro studies first identify possible candidates based on pharmacological activity, and then, in vivo (i.e., in animal or in human) studies further evaluate efficacy and safety. Later, additional human studies are conducted and then large-scale clinical trials prior to drug approval. A rule of thumb however is for every 250 compounds tested at these early stages ("preclinical research"), only one will make it to human use [4]. So, what are the chances cannabinoids become a COVID-19 therapy?

The van Breemen cannabinoid study suffers from similar limitations that other drug repurposing studies have encountered, including the low likelihood of replicating observed effects in the complex human body and the issue of realistic dosing. An illustration of these limitations when translating preclinical experiments to clinical relevance is readily available in the case of ivermectin. A study by Caly et al. [5] used in vitro experiments to confirm that ivermectin, an antiparasitic medication used for lice and roundworms in humans and prevention of heart- worms in dogs, blocked SARS-CoV-2. Clinical trials, observational studies, and meta-analyses of studies in humans provided mixed results for ivermectin as a potential COVID-19 therapy (prior to a cascade of falsified data admissions and retracted preprint articles) [6, 7]. Reliable evidence now demonstrates that ivermectin likely has no potential as a COVID-19 therapy [6]. Similar findings emerged from research evaluating hydroxychloroquine and azithromycin, with rigorously designed human studies concluding these regimens are not useful for preventing or treating SARS-CoV-2 infection and, in some cases, not being safe for use [8-12].

The common thread in much of this drug repurposing work is related to the dose. Looking at the in vitro ivermectin studies from a practical standpoint shows that the in vitro doses needed to block the virus were $>35$-fold higher than those used in humans [13]. In the human body, the amount of medication peaks sometime after administration then decreases over time, yet in vitro studies provide a consistently high dose. Thus, the stated concentrations achieved in human subjects represent the maximum amount of medication in the plasma after a dose, not a constantly available concentration [14]. The actual daily doses needed would be much higher to sustain this amount of drug in the plasma and therefore any therapeutic effect (Fig. 1). Similarly, our own calculation of azithromycin doses needed to replicate in vitro studies is at least $3 \times$ higher than those achieved with typical therapy [15]. With increasing doses come increasing safety concerns, and such high doses of any of these medications have never been studied in humans.

Our calculations from the van Breemen study also point to likely unachievable doses [1]. For CBDA, using several references from that paper, we estimate a dose of about $500-800 \mathrm{mg}$ is needed to reach the inhibitory concentrations stated by the authors. This concentration represents the peak, and CBDA reportedly has a short halflife of approximately $90 \mathrm{~min}$ [16], suggesting that this already high dose would be needed multiple times per day to maintain these inhibitory concentrations. Little evidence is available for CBDA in the human body, particularly at such high doses, so safety remains a key concern. For comparison, medical cannabinoids such as cannabidiol (CBD) and tetrahydrocannabinol (THC) are typically dosed around $20-25 \mathrm{mg}$, with maximum daily doses recommended of THC around $50 \mathrm{mg}$ and CBD doses of up to $20 \mathrm{mg} / \mathrm{kg} /$ day (used only for severe seizure disorders) [17-19]. The van Breemen study found that even higher doses of CBD and THC would be needed to achieve the same effect as CBDA and CBGA, making these com- 
mon cannabinoids also unlikely to be potential therapies [1]. This includes typical recreational use which involves doses hundreds of times lower than those found effective in the van Breemen study.

While inhibiting infection at high concentrations, the van Breemen findings are also restricted to a cell culture that does not consider the effects cannabinoids have on the human body as a complex system. The impact that these compounds have on a biological system are much harder to predict, even after demonstrating potential in blocking SARS-CoV-2 at an individual cell level. To illustrate, CBDA appears to have similar effects on the endocannabinoid system and other biological targets as CBD [20]. Thus, it is likely that CBDA will have a similar safety profile to high-dose CBD, which is only found in some prescription products [18]. There is reason for caution with $\mathrm{CBDA}$ and then because $\mathrm{CBDs}$ immunomodulatory effects are associated with increased mortality and impaired immune defenses $[18,21]$. Other cannabinoids, such as THC, are also associated with increased risk of infection [19]. Other safety concerns such as liver function, drowsiness, decreased appetite, and sleep disturbances are common but potentially manageable with high CBD doses $[18,22]$. On the other hand, CBD and CBDA may reduce inflammation, which is an important treatment target for serious SARS-CoV-2 infection [23]. However, other effective and well-studied compounds, such as corticosteroids, are available to achieve this goal.

Lastly, and perhaps most practically, CBDA is not a widely available cannabinoid, although it is generally present in Cannabis sativa [24]. CBDA converts to CBD when heated, so it will not be inhaled through smoking or similar routes of administration. Concentrated CBDA oils for oral use are available on the consumer or recreational markets in the USA for upwards of $\$ 100$ per $1,000 \mathrm{mg}$ as of 2022. Given the multiple doses per day of $500-800 \mathrm{mg}$ of CBDA needed, this potential therapy could cost upwards of $\$ 200$ or more per day without clear benefit.
While the van Breemen study is intriguing from a basic science perspective, it failed to convincingly make a case for the clinical potential of cannabinoids to prevent or treat SARS-CoV-2 infection. In vitro studies for drug repurposing are best contextualized based on achievable human doses, especially in the context of safety. We cannot conclude that cannabinoid doses found to be effective against SARS-CoV-2 during in vitro experiments are likely achievable. Cannabinoids, including CBDA and CBGA, are therefore not recommended for these purposes in clinical practice.

\section{Conflict of Interest Statement}

The authors have no conflicts of interest to declare.

\section{Funding Sources}

J.D.B. and A.J.G. are supported by the state of Florida appropriations to the Consortium for Medical Marijuana Clinical Outcomes Research (mmjoutcomes.org).

\section{Author Contributions}

J.D.B. conceptualized and drafted the work. A.J.G. revised the work. All the authors approved the final version.

\section{Editor Note}

Evidence in the context is part of the outreach effort of the Consortium for Medical Marijuana Clinical Outcomes Research to examine and discuss implications of research into cannabis and cannabinoids for clinical practice, thus providing a translational approach to these studies to make clear, concise, and actionable evidence available for clinicians and patients.

\section{References}

1 van Breemen $\mathrm{RB}$, Muchiri RN, Bates TA, Weinstein JB, Leier HC, Farley S, et al. Cannabinoids block cellular entry of SARS$\mathrm{CoV}-2$ and the emerging variants. J Nat Prod. 2022 Jan;85(1):176-84.

2 Khan SA, Al-Balushi K. Combating COVID-19: the role of drug repurposing and medicinal plants. J Infect Public Health. 2021 Apr;14(4):495-503.
3 Dotolo S, Marabotti A, Facchiano A, Tagliaferri R. A review on drug repurposing applicable to COVID-19. Brief Bioinform. 2021 Mar 22;22(2):726-41.

4 Harrer S, Shah P, Antony B, Hu J. Artificial intelligence for clinical trial design. Trends Pharmacol Sci. 2019 Aug;40(8):577-91.

5 Caly L, Druce JD, Catton MG, Jans DA, Wagstaff KM. The FDA-approved drug ivermectin inhibits the replication of SARS-CoV-2 in vitro. Antiviral Res. 2020;178:104787.
6 Lawrence JM, Meyerowitz-Katz G, Heathers JAJ, Brown NJL, Sheldrick KA. The lesson of ivermectin: meta-analyses based on summary data alone are inherently unreliable. Nat Med. 2021 Nov;27(11):1853-4.

7 Elgazzar A, Eltaweel A, Youssef SA, Hany B, Hafez M, Moussa H. Efficacy and safety of ivermectin for treatment and prophylaxis of COVID-19 pandemic. 2020. Preprint (Version 3) Available at: 
8 Rosenberg ES, Dufort EM, Udo T, Wilberschied LA, Kumar J, Tesoriero J, et al. Association of treatment with hydroxychloroquine or azithromycin with in-hospital mortality in patients with COVID-19 in New York state. JAMA. 2020 Jun 23;323(24):2493-502.

9 VouriSM, Thai TN, Winterstein AG. An evaluation of co-use of chloroquine or hydroxychloroquine plus azithromycin on cardiac outcomes: a pharmacoepidemiological study to inform use during the COVID19 pandemic. Res Social Adm Pharm. 2021 Jan;17(1):2012-7.

10 Gautret P, Lagier JC, Parola P, Hoang VT, Meddeb L, Mailhe M, et al. Hydroxychloroquine and azithromycin as a treatment of COVID-19: results of an open-label non-randomized clinical trial. Int $J$ Antimicrob Agents. 2020 Jul;56:105949.

11 Lane JCE, Weaver J, Kostka K, Duarte-Salles T, Abrahao MTF, Alghoul H, et al. Safety of hydroxychloroquine, alone and in combination with azithromycin, in light of rapid widespread use for COVID-19: a multinational, network cohort and self-controlled case series study. medRxiv. 2020. 20054551.

12 Sarayani A, Cicali B, Henriksen CH, Brown JD. Safety signals for QT prolongation or torsades de pointes associated with azithromycin with or without chloroquine or hydroxychloroquine. Res Social Adm Pharm. 2020;17(2): 483-6.
13 Schmith VD, Zhou JJ, Lohmer LRL. The approved dose of ivermectin alone is not the ideal dose for the treatment of COVID-19. Clin Pharmacol Ther. 2020 Oct;108(4):762-5.

14 Smith DA, Beaumont K, Maurer TS, Di L. Relevance of half-life in drug design. J Med Chem. 2018 May 24;61(10):4273-82.

15 Andreani J, Le Bideau M, Duflot I, Jardot P, Rolland C, Boxberger $\mathrm{M}$, et al. In vitro testing of combined hydroxychloroquine and azithromycin on SARS-CoV-2 shows synergistic effect. Microb Pathog. 2020 Aug;145: 104228.

16 Anderson LL, Low IK, Banister SD, McGregor IS, Arnold JC. Pharmacokinetics of phytocannabinoid acids and anticonvulsant effect of cannabidiolic acid in a mouse model of dravet syndrome. J Nat Prod. 2019 Nov 22; 82(11):3047-55.

17 Brown JD, Rivera Rivera KJ, Hernandez LYC, Doenges MR, Auchey I, Pham T, et al. Natural and synthetic cannabinoids: pharmacology, uses, adverse drug events, and drug interactions. J Clin Pharmacol. 2021 Aug;61 Suppl 2: S37-52.
18 Brown JD, Winterstein AG. Potential adverse drug events and drug-drug interactions with medical and consumer cannabidiol (CBD) use. J Clin Med. 2019 Jul 8;8(7):989.

19 Brown JD. Potential adverse drug events with tetrahydrocannabinol (THC) due to drugdrug interactions. J Clin Med. 2020;9(4):919.

20 Zagzoog A, Mohamed KA, Kim HJJ, Kim ED, Frank CS, Black T, et al. In vitro and in vivo pharmacological activity of minor cannabinoids isolated from Cannabis sativa. Sci Rep. 2020 Nov 23;10(1):20405.

21 Reiss CS. Cannabinoids and viral infections. Pharmaceuticals. 2010 Jun 1;3(6):1873-86.

22 EPIDIOLEX (cannabidiol) Prescribing information. https://www.epidiolex.com/sites/default/files/EPIDIOLEX_Full_Prescribing_Information.pdf (accessed June 9, 2019).

23 Brown JD. Cannabidiol as prophylaxis for SARS-CoV-2 and COVID-19? Unfounded claims versus potential risks of medications during the pandemic. Res Social Adm Pharm. 2021;17(1):2053

24 Aizpurua-Olaizola O, Soydaner U, Öztürk E, Schibano D, Simsir Y, Navarro P, et al. Evolution of the cannabinoid and terpene content during the growth of cannabis sativa plants from different chemotypes. J Nat Prod. 2016 Feb 26;79(2):324-31. 